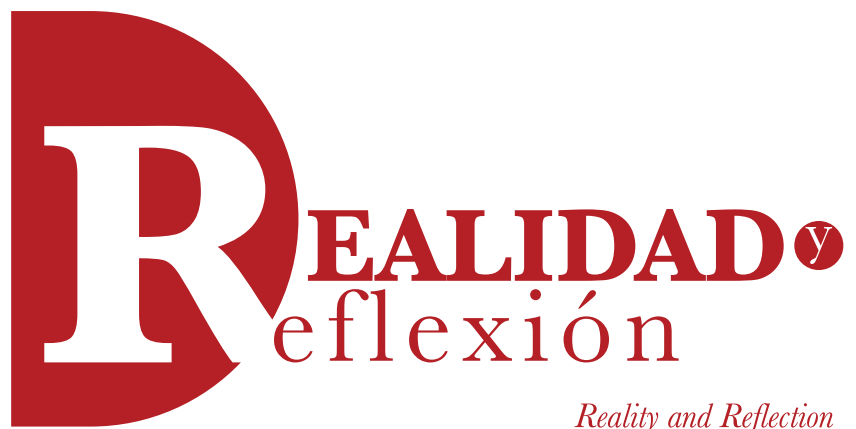

ISSN 1992-6510

Año 16, N 43, San Salvador, El Salvador, Centroamérica. Revista Semestral Enero-Junio 2016

YeAR 16, N 43, SAN SALVADOR, EL SALVADOR, CENTRAL AmERICA. SEMESTRAL JOURNAL JANUARY-June 2016

\title{
Descripción de la Organización Virtual
}

\author{
Virtual Organizations
}

\author{
Víctor Cuchillac \\ Ingeniero en Electrónica por la Universidad Don Bosco. \\ Maestro en Informática Aplicada en Redes por la Universidad Francisco Gavidia \\ Investigador para el área de Ingeniería y Tecnología, ICTI-UFG \\ vcuchillac@ufg.edu.sv
}

\section{RESUMEN}

Este artículo tiene como finalidad resumir y difundir el marco conceptual de las "organizaciones virtuales", bajo una óptica estratégica orientada a la competitividad empresarial, haciendo énfasis en las características, las ventajas y las desventajas de estas. Además, se aborda de forma sucinta el perfil del gerente tanto administrativo como de tecnología, que permita a una organización migrar parcial o completamente sus procesos a la nube. Tecnológicamente muchas organizaciones de la región centroamericana están inmersas en la virtualización y tratan de aprovechar sus beneficios, pero como la tecnología y la dinámica de las empresas avanza galopantemente a otro ritmo, es necesario conocer y difundir la tendencia actual del modelo de la "organización virtual". Es por ello, necesario que, tanto los formadores como nuevos profesionales, conozcan de esta tendencia de forma que al vincularse con el mundo productivo sean agentes de cambio que orienten y lideren los movimientos técnicos estratégicos.

Palabras clave: organizaciones virtuales, CAPEX, OPEX, IaaS, PaaS, PaaS.

\section{ABSTRACT}

This article aims to summarize and disseminate the conceptual framework of "Virtual Organizations" under a strategic perspective oriented business competitiveness, emphasizing the characteristics, advantages and disadvantages of these. In addition, succinctly talk about the profile for administrative manager and IT manager, allowing an organization partially or completely migrate their processes to the cloud. Technologically many organizations in Central America are engaged in virtualization and try to reap its benefits, but as technology and business dynamics advances so fast, it is necessary to know and disseminate the current trend model of "virtual organization". It is therefore necessary that both trainers and new professionals are aware of this trend so that the link with the productive world are agents of change to guide and lead the strategic technical desitions.

Keywords: virtual organizations, CAPEX, OPEX, IaaS, PaaS, PaaS. 


\section{Introducción}

Con el desarrollo de la tecnología y el advenimiento de los servicios en la nube, las empresas cada día poseen las condiciones para tener toda la infraestructura tecnológica de forma virtual y desarrollar los procesos de la empresa de forma remota o tercerizada; es por ello que el concepto de "organizaciones virtuales" es cada vez más real, y no solo abarca a la tecnología utilizada, sino también es un concepto de distribución de funciones y tareas dentro de la organización; es decir, los procesos y las tareas pueden ser realizados remotamente por individuos y sistemas electrónicos que pertenezcan a otra organización. La flexibilidad para acoplarse a los nuevos cambios y la reducción de costos son solo un par de elementos que potencian a las organizaciones virtuales. Así que, los gerentes administrativos y gerentes de tecnología deben estar preparados para acoplarse a los nuevos retos propuestos por la tendencia de la virtualización de las organizaciones; de forma que, la empresa que lideran pueda tomar las ventajas que le permitan crecer, y protegerse del nuevo esquema de operación.

\section{Marco conceptual}

La definición del paradigma organización virtual es más que la unión de sus sustantivos e involucra una serie de definiciones que permiten inferir el reciente concepto organizacional, en el artículo "Organizaciones Virtuales: De la integración a la desintegración integrada” (Araya Guzmán \& Criado Fernández, 2004, pág. 32), cuyos autores han recopilado una serie de definiciones muy completas que puntualizan la particularidad de este tipo de organización. Entre estas definiciones se mencionan las siguientes.
"Es la estructura organizativa que, incluyendo componentes de una o varias empresas, se ha configurado en torno a un conjunto de competencias esenciales, fomentando la cooperación a través de un uso adecuado de las tecnologías de información y las comunicaciones, y cuya finalidad básica consiste en la realización de procesos de negocio orientados a la creación de valor para todos" (Martínez, M. y De Pablos, C., 2001).

"Es una colección temporal o permanente de individuos dispersos geográficamente, grupos - unidades organizativas que no pertenecen a la misma organización, u organizaciones que dependen de enlaces electrónicos para completar el proceso de producción" (Bob Travica, 1997).

"Es una red temporal de organizaciones independientes vinculadas por tecnologias de la Información para compartir riesgos, costes $y$ acceso a otros mercados. Las organizaciones se unen de forma rápida para explotar una oportunidad especifica, y a continuación se dispersarán” (Byrne, J., 1993).

"Esuna organización en red, queestá estructurada y gestionada de una forma tal que opera 'codo a codo' con clientes y otros participantes, como una organización identificable y completa" (Aken, J., Hop, L. y Post, G., 1997).

En función de lo anterior se observa que las organizaciones virtuales siguen manteniendo muchas de las características de las organizaciones tradicionales; sin embargo, también poseen características muy similares a los servicios informáticos virtuales, los cuales se ejecutan muchas veces por proveedores externos 
(outsourcing), una administración y una estructura muy flexible, la cual se ajusta a las necesidades con el fin de adaptarse al mercado, a la naturaleza para la cual ha sido creada y para la efectividad en el logro de los objetivos.

En una forma más conceptual, una organización virtual posee un funcionamiento sencillo de exponer, "diferentes organizaciones vinculadas entre sí, realizan de manera coordinada distintas actividades para producir de forma combinada un producto $\mathrm{u}$ ofrecer un servicio. La vinculación es generalmente temporal y se establece por sistemas de información interorganizacionales conectados por una red de datos” (Araya Guzmán \& Criado Fernández, 2004, pág. 119).

\section{A. Características de las organizaciones virtuales}

Algunas características de las organizaciones virtuales (Araya Guzmán \& Criado Fernández, 2004) son estas:

- Uso muy fuerte de las $\mathrm{TIC}^{1}$ para los procesos principales de la organización, los aplicativos y los sistemas para la gestión, las comunicaciones, etc.

- La temporalidad, ya que muchas se enfocan en el desarrollo de proyectos o productos puntuales, por lo cual una vez terminado dicho proyecto el personal contratado finaliza las funciones asignadas.

- Son estructuras de gobierno basadas en la confianza.

1 Acrónimo para Tecnologías de la Información y la Comunicación.
- Formadas por unidades legalmente independientes.

- Requieren de aprendizaje por parte de la organización y orientación a la adaptación.

\section{B. Ventajas y desventajas de las organizaciones virtuales}

De una manera sintetizada se amplían los conceptos enumerados por Carol Guerrero en relación a las ventajas y desventajas de las organizaciones virtuales. (Guerrero, 2013)

A continuación, se listan las ventajas más relevantes:

A continuación, se listan las ventajas más relevantes:

- Son flexibles. Ya que se acomodan a las necesidades de la empresa, esta flexibilidad aplica a la cantidad de empleados que se requiere y los servicios de gestión y sistemas que se necesitan. Por ejemplo, empresas que desarrollan aplicaciones tipo start$u p,{ }^{2}$ videojuegos, comercio electrónico, aplicaciones para móviles, etc.

\section{- Difusas en cuanto a la estructura organizativa.} Los límites de las funciones no son claros, ante un fallo o deducción de responsabilidades es más difícil encontrar a quién y cómo se debe proceder de forma efectiva. Algunas funciones pueden estar contenidas en dos empresas diferentes que han sido contratadas.

2 Las Empresas start-up son emprendimientos temporales; es decir, que poseen una historia de funcionamiento limitado, que se distinguen de otros tipos tradiciones porque poseen un perfil de riesgo augurando en contrapartida un gran retorno o utilidad financiera, con perspectivas de crecimiento y diversificación. 
- Rápidas. Debido a la flexibilidad de su estructura y capacidad para administrar los recursos. También esta rapidez se puede observar en el tiempo que toma crear nuevos departamentos o modificar su tamaño en función de las necesidades del proyecto o producto que se está desarrollando.

\section{- Disponibilidad de operación las 24 horas del} día. Al tener "servicios en la nube", ${ }^{3}$ los usuarios pueden acceder a ellos desde cualquier lugar y a cualquier hora; un ejemplo de esto son las tiendas en línea para envío de arreglos florales, bancos, servicios de impuestos, etc.

\section{- Reducción en la inversión tecnológica} debido a la reducción en los montos del $\mathrm{CAPEX}^{4}$ como del OPEX. ${ }^{5}$ En el primer caso porque no hay necesidad de invertir completamente en la infraestructura computacional, generalmente compuesta por: equipos informáticos para servidores, las instalaciones físicas y eléctricas para la red de datos, los equipos terminales para los usuarios y el soporte, las licencias de software, entre otros; ya que, los servicios de la empresa pueden ser ejecutados por "servicios en la nube o cloud computing". Para el segundo tipo de gastos, también existe reducción pues no es necesario la contratación de un gran departamento de informática propio, la

3 Son los servicios que se rentan a otras empresas, en donde el acceso es por la Internet.

4 CAPEX del inglés "Capital Expenditure", (Gastos de capital), se refiere a los montos de dinero gastado en la adquisición o renovación de los bienes de capital de una organización.

5 OPEX del inglés "Operating expense”, (Gastos de operacionales), es el importe de los costos permanentes para el funcionamiento de un producto, negocio o sistema. contratación tercerizada del soporte técnico, los montos por prestaciones en empleados, etc. Si los montos del CAPEX se reducen dramáticamente existirá un valor de OPEX que se incrementará (ver nota) ${ }^{6}$, en especial el uso del acceso a internet. Reducir los gastos de operación en una empresa, puede ser la diferencia entre la rentabilidad o el cierre de operaciones.

Como todo modelo o sistema, en contraparte de los beneficios, existen desventajas que deben ser consideradas, y en ese sentido aquí se amplían brevemente las desventajas listadas por Carol Guerrero (Guerrero, 2013).

- La potencial pérdida del know-how. Es el conocimiento colectivo y valioso que se incrementa a medida que las organizaciones logran las metas iniciales e incorporan procesos de mejora continua, dicho conocimiento y la experiencia colectiva se ven perdidos si para el siguiente proyecto o producto, la empresa no dispone del mismo personal porque ya no se encuentra disponible. Además, otro aspecto negativo es que el know-how contenido en el personal que fue contratado temporalmente o fue provisto por tercerización, puede ser empleado en actividades realizadas por la competencia.

- El valor agregado se disipa, a medida que intervienen más sujetos que no forman

\footnotetext{
6 En el caso que se virtualicen los servicios con Cluod Computing, puede darse el escenario que los montos del CAPEX caigan dramáticamente y que los costos del OPEX se incrementen, pero al final de la operación el ahorro es significativo, y esto se nota en cómo se han diversificado los servicios de virtualización y Cloud Computing, de los cuales se abordará posteriormente.
} 
parte de la empresa en el desarrollo de las actividades claves, es más difícil mantener la mística de valor agregado.

- Se requieren directivos con un perfil de liderazgo orientado a la virtualización que contengan entre varias características las siguientes: capacidad para la planificación con recursos locales y remotos, confianza en la delegación en personas que nunca han visto, motivación a grupos de individuos cuya relación no es personal, y saber aplicar procedimientos de control de manera que se logren los objetivos deseados.

- La identificación de los empleados con la organización es mucho menor que en los modelos tradicionales. Esto, debido a que un teletrabajador puede realizar otras funciones dentro de su período laboral, y en el caso de la tercerización (outsourcing) las empresas o personas contratadas tienen más de un cliente a quien atender.

- Reducción de un protector fiscal. Este elemento es muy propio de sistemas fiscales en donde las empresas pueden obtener un "escudo fiscal" para sus operaciones, ya que pueden apoyarse en depreciaciones para reducir la carga fiscal.

Para ejemplarizar de forma sucinta las organizaciones virtuales se comentará brevemente acerca de empresas comerciales muy populares. La primera es Amazon ${ }^{7}$ la cual

\footnotetext{
7 Amazon Inc. es una compañía estadounidense de Comercio electrónico y Servicios de Cloud Computing o computación en la nube (infraestructura, plataforma y servicios), la empresa posee la sede en Seattle.
}

vende una diversidad de productos en línea. En Amazon casi la totalidad de los procesos de gestión interna se realiza en línea. La atención al cliente se hace por medio de un call center que atiende a los usuarios por medio del chat o por llamada telefónica tradicional y en varios idiomas, en donde los operadores se encuentran en diferentes partes de los Estados Unidos, esto debido a que existen muy pocas tiendas locales tanto en Estados Unidos como en otros países. Los pagos como es sabido, se realizan por cargos en tarjeta de crédito, débito o por empresas intermediarias como Paypal (la cual es otra organización virtual orientada a servicios financieros en línea). Las entregas se hacen por el correo nacional estadounidense o por empresas que el usuario puede seleccionar a la hora de aplicar la orden de compra, (electrónicamente es posible que el usuario controle dónde se entregue el producto). También Amazon posee servicios de entretenimiento digital, ${ }^{8}$ en cuyo caso inyecta tráfico proveniente de canales privados o públicos, brindando así la transmisión de películas, series, u otro tipo de broadcast. También Amazon posee servicios de notificación. Y por si no fuera poco, Amazon ofrece los servicios de cloud computing a empresas. ${ }^{9}$

$\mathrm{Si}$ bien es cierto que las organizaciones virtuales cada vez son más comunes, no significa que las empresas tradicionales deban migrar a este modelo, ya que las primeras surgen en concordancia con los nuevos retos y las características de los tiempos de la

\footnotetext{
8 Para más información del servicio Prime se puede consultar el contenido disponible en el sitio Web http://www.amazon.com/ Amazon-Prime-One-Year-Membership/dp/B00DBYBNEE

9 Más información en español se encuentra disponible en el sitio web https://aws.amazon.com/es/
} 
gestión postmoderna (Gentilin, 2015). Las organizaciones virtuales deben considerarse como otro tipo más dentro de las organizaciones. Las empresas pueden crear servicios híbridos de forma que puedan aprovechar los beneficios de las organizaciones virtuales antes descritas.

Pero las organizaciones virtuales no solo son de tamaños inmensurables, ya que también existen empresas pequeñas que han surgido como startup, por ejemplo Sculpeo ${ }^{10}$ la cual es una empresa dedicada a la impresión en $3 \mathrm{D}^{11}$ en dónde los usuarios, vía electrónica, envían sus planos o archivos para recibir a través del correo tradicional las piezas de los planos que se han enviado. Esta empresa contrata los servicios de pago en línea con operadores de tarjetas de crédito, contrata a operarios locales que se encargan de realizar la impresión, establece compras en líneas de los proveedores de los materiales para la impresión (polímeros, resinas, polvo de metales, etc.), compra porque no desarrolla ella misma las impresoras 3D con proveedores que venden en línea sus productos y envía por correo las piezas a los usuarios.

\section{Componentes más relevantes de las organi- zaciones virtuales}

Para comprender los elementos claves de una organización virtual que opera con teletrabajadores, servicios en outsourcing y uso extensivo de TIC como podrían ser los call center, es necesario ampliar los elementos que definen a dichas organizaciones virtuales.

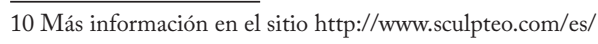

11 Impresión 3D es la tecnología que permite crear de forma económica objetos generalmente en polímeros, metal, resinas entre otros.

\section{El entorno tecnológico para las empresas virtuales}

La característica que diferencia a las organizaciones virtuales de otros tipos de organizaciones es el uso preponderante de las TIC (Tecnologías de la Información y Comunicación) para proveer la interacción de individuos de forma remota. Muchas organizaciones emplean las TIC para el desarrollo de los procesos; pero lo hacen adsorbiendo los costos de poseer la infraestructura de servicios y dando en outsourcing algunos de ellos. Sin embargo, cuando las empresas se encargan de ejecutar proyectos específicos, los costos por tecnología, salarios y otros son muy significativos para la rentabilidad; es por ello, que es común ver organizaciones virtuales consumiendo servicios en la nube, que les permiten escalar ${ }^{12}$ o reducir los costos en función del uso.

Con la era del conocimiento y un galopante desarrollo de tecnologías, las empresas pueden consumir servicios tecnológicos de forma remota de otras empresas especializadas en proporcionar dichos servicios. Este reciente paradigma se conoce como computación en la nube "cloud computing”. Según el NIST ${ }^{13}$ existen tres modelos de servicios disponibles para las empresas: SaaS, PasS e IaaS. Acorde a la clasificación del NIST (NIST, 2010), el primer modelo es el SaaS "Software como Servicio", el cual permite a las empresas consumir los servicios específicos sin preocuparse por los costos relacionados con los equipos y las configuraciones. La desventaja de

12 Por escalabilidad se debe entender la capacidad de crecer o incrementar la infraestructura tecnológica o los aplicativos informáticos de una organización.

13 National Institute of Standards and Technology. 
este servicio consiste en que solo se personalizan las características del mismo; son ejemplos: el correo electrónico (Outlook 365 de Microsoft Corporativo, Gmail Enterprise, etc.); los servicios de VoIP (Voz sobre IP), o telefonía por red de datos (Skype empresarial, Google Hangouts, VoxOx, etc).

Pero las empresas poseen aplicativos específicos como, por ejemplo: sistemas de inventario, sistemas ad-hoc de producción, sistemas contables, etc. En los cuales se necesita el desarrollo o la modificación profunda del software por parte de la organización. Es aquí donde el segundo modelo PaaS - Plataforma como Servicio, presenta las condiciones para colocar y darle soporte a los sistemas de las empresas. Los servicios de desarrollo que ofrece Azure de la empresa Microsoft son un ejemplo de este tipo de modelo, en donde las empresas rentan el servicio de la base de datos, plataforma de desarrollo .Net y los de publicación y capa de proceso, utilizando y configurando los recursos disponibles. E1 último modelo de servicio tipificado por el NIST corresponde a IaaS - "Infraestructura como Servicio", en el cual la empresa puede crear todos los servidores que se desee; es decir, que ya una organización no necesita más que el acceso a la Internet y el pago por el uso de los servidores, de forma que pueda ejecutar todo el software que se encuentra instalado localmente en los equipos virtualizados contratados.

Es así como las organizaciones, tanto privadas como públicas en términos tecnológicos poseen las condiciones para trasladar parte o todos sus equipos al Internet (nube), reduciendo con ello los costos e incrementando la rentabilidad, la visibilidad, la jornada (24 horas durante 7 días por año). Así mismo, este modelo permite a empresas nuevas comenzar a operar de una forma mucho más económica que si lo hicieran de manera tradicional, ya que requerirían más tiempo para iniciar operaciones debido a la adecuación del local, instalación de la red, adquisición de los equipos, contratación del personal, instalación y mantenimiento de los aplicativos, entre otros.

\section{La gestión dentro de las organizaciones virtuales}

En un entorno virtual donde los subordinados se interrelacionan por medio de sofisticados sistemas de comunicación, pero de fácil uso y acceso, el gerente debe tener las competencias que le permitan el logro de las metas de la empresa, mantener la motivación y la efectividad (eficacia y eficiencia) de los diferentes actores, ya sean tanto propios o contratados, como outsourcing por ejemplo. Lo cual no es nuevo, pero el nivel de confianza que se requiere en las organizaciones virtuales es mucho mayor; ya que algunos autores, expertos en la administración, han sugerido ciertos principios que deben tener los líderes de las organizaciones virtuales en relación con la confianza en los subalternos; por ejemplo, Charles Handy propone lo siguiente (Lawrence, 2003):

- No estar confiado permanentemente en las acciones de los subordinados virtuales. Es decir, contratar los servicios de empresas o personas conocidas permite tener cierta confianza, que permite reducir el riego retrasos y productosservicios que no cumplen las especificaciones.

- Es necesario delegar en los individuos que realizarán las tareas, confiando en la idoneidad del perfil de ellos para el logro de las actividades y metas de la empresa. 
- Si existe alguna falla por parte de los subordinados, dichos subordinados o proveedores deben retirarse de la empresa; ya que, de lo contrario no existirá control efectivo por parte del líder o gerente.

- La confianza requiere de contacto frecuente, el contacto estará orientado en el logro de los objetivos y al desarrollo de las actividades.

El trabajo de los gerentes de las organizaciones siempre ha estado circunscrito a horarios no bien delimitados;yparauna organización virtualdónde los horarios de trabajo de los miembros podrían distar muchos debido a las diferencias horarias, la capacidad de organización y seguimiento será mayor; también las competencias para el uso de las tecnologías serán mayores; por ejemplo, el uso de la videoconferencia, el uso de las plataformas de trabajo colaborativo, el uso de los dispositivos móviles para las comunicaciones unificadas, etc.

\section{El tipo de organización}

En las organizaciones virtuales no existe una estructura propia, ya que estas pueden adoptar diferentes composiciones en función de la estructura que mejor se adapte al desarrollo de las metas de la organización; en este sentido, se puede hablar de modelos híbridos. Lo que sí no es admisible es la burocracia, la inflexibilidad es más bien un caos controlado, algo un tanto caórdico, ${ }^{14}$ si no considere los macroprocesos y procesos brevemente descritos anteriormente para las ventas de Amazon.

14 Entendiéndose por caórdica al tipo de organización que se auto regula, se reordena de una forma compleja no lineal que permiten coexistir el orden y el caos, logrando a su vez la cooperación y la competencia, y la continuidad y la creatividad de sus miembros.
Muchos autores han tratado de clasificar o describir las características principales de las organizaciones virtuales; sin embargo, no existe una clasificación estandarizada. Después de consultar algunas propuestas, la clasificación realizada por Mariano Getillin presenta rasgos que pueden ser muy fáciles de identificar en organizaciones en la región centroamericana (Gentilin, 2015, pág. 17) La clasificación de las empresas se presenta a continuación. (Ver Cuadro n. ${ }^{\circ} 1$ )

\section{Conclusiones}

Debido al cambiante mercado y al entorno tecnológico, las organizaciones que puedan adaptarse a estos cambios tienen mayor probabilidad de éxito, y no es necesario migrar directamente de un esquema tradicional a un esquema virtual. Las empresas privadas pueden ejecutar procesos menos susceptibles e ir migrando, si con ello se logra el cumplimiento de las metas de una manera más eficaz y eficiente. Obviamente, para las organizaciones públicas esta migración no es expedita y requerirá de una serie de pasos estratégicos comenzando por las tareas de reducción de costos por inversiones en adecuación, instalación de la red de datos, capacitación especificada al personal o contratación de los servicios a otras compañías.

La virtualización de procesos en una organización presenta opciones que reducen los costos del CAPEX y del OPEX, pero también hay que considerar aquellos aspectos muy propios de la empresa. Como se expuso anteriormente el know how, como activo intangible, es algo que debe ser considerado y resguardado en aquellas organizaciones que dependan de este capital como 


\section{Cuadro n. ${ }^{\circ} 1$}

\section{La clasificación de las empresas}

\begin{tabular}{|c|c|c|c|}
\hline Categorías & $\begin{array}{l}\text { Organización con grados } \\
\text { de virtualidad }\end{array}$ & $\begin{array}{c}\text { Organización virtual } \\
\text { temporal }\end{array}$ & Organización virtual \\
\hline Estructura & $\begin{array}{c}\text { Formal o informal, jerárquica } \\
\text { o plana, rígida o flexible. }\end{array}$ & Formal o informal, flexible. & Formal o informal, flexible. \\
\hline $\begin{array}{c}\text { Tipo de acoplamiento } \\
\text { estructural }\end{array}$ & Estrecho o flojo & Flojo & Flojo \\
\hline Fronteras & Relativamente identificables & $\begin{array}{l}\text { Poco identificables o } \\
\text { inexistentes }\end{array}$ & $\begin{array}{l}\text { Poco identificables o } \\
\text { inexistentes }\end{array}$ \\
\hline Tipo de tecnología (hard) & $\begin{array}{c}\text { Grandes o pequeñas } \\
\text { maquinarias o dispositivos } \\
\text { tecnológicos }\end{array}$ & $\begin{array}{l}\text { Pequeños dispositivos } \\
\text { tecnológicos }\end{array}$ & $\begin{array}{l}\text { Pequeños dispositivos } \\
\text { tecnológicos }\end{array}$ \\
\hline Tipo de tecnología (soft) & Sencillos o complejos & $\begin{array}{c}\text { Complejos, integrados o } \\
\text { virtuales }\end{array}$ & $\begin{array}{c}\text { Complejos, integrados o } \\
\text { virtuales }\end{array}$ \\
\hline Aprendizaje & Mínimo o estimulado & Relativo o estimulado & Constante y estimulado \\
\hline Formas de control & $\begin{array}{c}\text { Control externo o } \\
\text { autocontrol }\end{array}$ & Autocontrol o confianza & Autocontrol o confianza \\
\hline $\begin{array}{l}\text { Compromiso con la } \\
\text { organización }\end{array}$ & Mínimo o alto & Alto con el proyecto & Alto con la organización \\
\hline Tipo de relacionamientos & Formales o informales & Formales o informales & $\begin{array}{l}\text { Menos formales o mas } \\
\text { formales }\end{array}$ \\
\hline Motivación & $\begin{array}{c}\text { Economía, simbólica o } \\
\text { discursiva }\end{array}$ & $\begin{array}{c}\text { Economía, simbólica o } \\
\text { discursiva }\end{array}$ & $\begin{array}{c}\text { Economía, simbólica o } \\
\text { discursiva }\end{array}$ \\
\hline Producción & $\begin{array}{l}\text { En masa o especializada en } \\
\text { cantidad o calidad }\end{array}$ & Especializada y de calidad & $\begin{array}{c}\text { En masa o especializada en } \\
\text { calidad }\end{array}$ \\
\hline Forma de producción & Automatizada o artesanal & Automatizada & Automatizada \\
\hline Tipo de trabajador & Especializado o polivalente & Especializado o polivalente & Especializado o polivalente \\
\hline Grado de integración & Individual o en equipo & Equipos virtuales & $\begin{array}{c}\text { Individual o en equipo } \\
\text { virtual }\end{array}$ \\
\hline Niveles de utilidad & $\begin{array}{c}\text { Altos o bajos y de corto o de } \\
\text { largo plazo }\end{array}$ & Altos y de corto plazo & Altos y de largo plazo \\
\hline Ambiente y mercado & $\begin{array}{c}\text { Estable o polivalente } \\
\text { Restringido o en expansión } \\
\text { Estandarizado o diversificado }\end{array}$ & Polivalente o especializado & $\begin{array}{c}\text { Polivalente, especializado o } \\
\text { en expansión }\end{array}$ \\
\hline $\begin{array}{l}\text { Relacionados con los } \\
\text { proveedores }\end{array}$ & $\begin{array}{l}\text { Competencia estrecha de } \\
\text { corto o largo plazo }\end{array}$ & $\begin{array}{c}\text { Estrecha y alianza } \\
\text { Estratégica o virtual }\end{array}$ & $\begin{array}{l}\text { Estrecha y alianza } \\
\text { Estratégica virtual }\end{array}$ \\
\hline Relación con los clientes & Presencial y virtual & Presencial o virtual & Virtual \\
\hline $\begin{array}{c}\text { Concepción de la } \\
\text { organización }\end{array}$ & $\begin{array}{c}\text { Mecánica, orgánica o } \\
\text { cultural }\end{array}$ & $\begin{array}{l}\text { Orgánica, cultural y } \\
\text { ultraflexible }\end{array}$ & $\begin{array}{l}\text { Orgánica, cultural y } \\
\text { untraflexible }\end{array}$ \\
\hline $\begin{array}{c}\text { Forma de interacción entre } \\
\text { los miembros }\end{array}$ & Presencial y virtual & Virtual & Virtual \\
\hline Estilos de comunicación & $\begin{array}{l}\text { Formal o informal } \\
\text { Vertical o lateral } \\
\text { Presencial o virtual }\end{array}$ & $\begin{array}{l}\text { Formal o informal } \\
\text { Vertical o lateral } \\
\text { Virtual }\end{array}$ & $\begin{array}{l}\text { Predominantemente } \\
\text { informal, lateral o virtual }\end{array}$ \\
\hline
\end{tabular}


ventaja estratégica. También se debe considerar los riesgos y aspectos legales que conlleva tener la información financiera de la empresa en equipos ubicados en la nube. Las instituciones formadoras de profesionales como las universidades y los centros de habilitación laboral deben incluir en sus programas de estudio las características de la nueva tenencia del consumo de servicios por la computación en la nube. Así, los conocimientos, habilidades y actitudes permitirán a los nuevos profesionales incluirse como proveedores de servicios a organizaciones virtualizadas, poder crear start-up y dirigir instituciones en donde todos los servicios y relaciones se realizan de forma virtual; es decir, no solo desarrollar las competencias técnicas y fomentar el emprendimiento por proyectos, sino que es necesario desarrollar un perfil ya sea para la dirección o brindar servicios a este tipo de organizaciones.

El personal técnico puede verse beneficiado con la incursión en temas de administración, como estructura de las organizaciones, definición de políticas y estrategias claves, medición de los indicadores clave $\mathrm{KPI},{ }^{15}$ entre otros, porque la tecnología debe apoyar y mejorar los procesos de las organizaciones, y no la organización debe apegarse a la tecnología que se utiliza. Así, un directivo técnico puede comprender y anticiparse en su rol (implementador y proveedor de soporte a otros procesos), a las adaptaciones que debe realizar la organización para ser competitiva e innovadora. Para el personal técnico de sistemas no solo le es necesario poder montar estructuras virtuales (servidores, plataformas en línea, Accesos a redes privadas virtuales "VPN", etc.), sino entender cómo toda la organización puede

15 KPI Acrónimo inglés para "Key Performance Indicators", que en español se interpreta como "Indicadores clave del desempeño". volverse virtual, así también sus cualidades de liderazgo deberán ser modificadas para interactuar con las personas o los servicios externos y dejar esa característica del personal de sistemas en restringir todo y ser poseedor de los accesos a la información, obviamente sin exponer la seguridad de la información y la eficiencia de los procesos.

\section{Referencias Bibliográficas}

Araya Guzmán, S., \& Criado Fernández, M. (2004). Organizaciones virtuales de la integración a la desintegración integrada. Revista Ingeniería industrial, 31-45.

Criado Fernández, M. D., Arroyo Barrigüete, J. L., \& López Sánchez, J. I. (2005). ORGANIZACIONES VIRTUALES Y REDES NEURONALES. ALGUNAS SIMILITUDES. Estudios Gerenciales, 117-125.

Gentilin, M. (2015). La Organización Virtual: caracterización, diferenciación y conceptualización. Estudios Interdisciplinarios de la Organización, 2-24.

Guerrero, C. (17 de Agosto de 2013). Administración de Empresas Virtuales. Obtenido de La organización virtual: https:// carolromero.wordpress.com/2013/08/17/1aorganizacion-virtual/

Lawrence, F. (Octubre de 2003). The Paradox of Charles Handy. Obtenido de http://www. strategy-business.com/article/03309?gko=f3861

NIST. (15 de Noviembre de 2010). NIST Cloud Computing Program. Obtenido de http://nvlpubs.nist.gov/nistpubs/Legacy/SP/ nistspecialpublication800-145.pdf 